\title{
ARTIGOS
}

\section{MONITORIZAÇÃO DO PROGRESSO DO ALUNO NA IDENTIFICAÇÃO DE RISCO NA LEITURA}

PAULA MARISA FORTUNATO VAZ • ANA PAULA LOUÇÃO MARTINS • LUÍS DE MIRANDA CORREIA

\section{RESUMO}

Este estudo tem por finalidade analisar o uso da monitorização com base no currículo como sistema escolar de identificação de alunos em risco na leitura, no contexto de um modelo educativo baseado no grau de resposta à intervenção. Realizou-se uma investigação quantitativa em que participaram todos os alunos do $3^{\circ}$ ano (82 alunos) de um agrupamento de escolas do norte de Portugal. 0 tratamento de dados foi feito com recurso à estatística descritiva e inferencial. Destacam-se, entre outras conclusões, a existência de dez alunos em risco na leitura durante todo o ano letivo e o facto de, no final do $3^{\circ}$ ano de escolaridade, a média de resultados dos alunos que nunca estiveram em risco ser superior ao dobro da média de resultados dos alunos que estiveram em risco todo o ano.

\section{LEITURA • PORTUGAL・PESQUISA QUANTITATIVA • DIFICULDADE DE APRENDIZAGEM}

\section{MONITORING STUDENT PROGRESS IN IDENTIFYING RISK IN READING}

\section{ABSTRACT}

This study aims to analyze the use of curriculum-based monitoring as a school system to identify students at risk in reading, in the context of an educational model based on the degree of response to intervention. A quantitative investigation was conducted with all third grade students (82 students) from a group of schools in the north of Portugal. Data processing was done using descriptive and inferential statistics. Among the conclusions, the following stand out: the existence of ten students at risk in reading throughout the school year and the fact that, at the end of the third year of schooling, the mean results of students who were never at risk was more than double the mean results of students who were at risk for the entire year. 
RÉSUMÉ

Cette étude a pour but d'analyser l'usage du contôle ayant pour base le coursus comme système scolaire d'identification des élèves présentant des risques dans la lecture, dans le contexte d'un modèle éducatif fondé sur le degrée de réponse à l'intervention. On a fait une investigation quantitative à laquelle ont participé tous les élèves de CM1 (82 élèves) d'un groupement d'écoles du nord du Portugal. Le traitement des données a été fait à l'aide de la statistique descriptive et inférentielle. On souligne, entre autres conclusions, l'existence de dix élèves en risque dans la lecture pendant toute l'année scolaire et le fait qu'à la fin de la troisièmme année de scolarité, la moyenne des résultats des élèves n'ayant jamais été en risque soit supérieur au double de la moyenne des résultats des élèves qui ont été en risque toute l'année.

\section{LECTURE • PORTUGAL ・ RECHERCHE QUANTITATIVE •}

DIFFICULTÉ DE L’APPRENTISSAGE

\section{MONITOREO DEL PROGRESO DEL ALUMNO EN LA IDENTIFICACIÓN DE RIESGO EN LA LECTURA}

RESUMEN

Este estudio tiene la finalidad de analizar el uso del monitoreo del currículo como sistema escolar de identificación de alumnos en riesgo en la lectura, en el marco de un modelo educativo basado en el grado de respuesta a la intervención. Se llevó a cabo una investigación cuantitativa en la que participaron todos los alumnos del 3er año (82 alumnos) de un agrupamiento de escuelas del norte de Portugal. El tratamiento de datos se efectuó por medio de la estadística descriptiva e de inferencias. Se destacan, entre otras conclusiones, la existencia de diez alumnos en riesgo en la lectura durante todo el año lectivo y el hecho de que, al final del 3er año de escolaridad, el promedio de resultados de los alumnos que nunca estuvieron en riesgo era superior al doble del promedio de resultados de los alumnos que estuvieron en riesgo todo el año. 


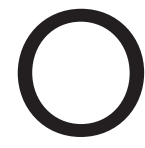

MODELO DE RESPOSTA À INTERVENÇÃO - MRI -, DESCRITO POR FLETCHER E VAUGHN (2009),

Johnson et al. (2006) e pelo National Joint Committee on Learning Disabilities - NJCLD (2005), é um modelo educativo no qual é delineado e implementado, ao longo de diferentes níveis, para os alunos com dificuldades académicas e de comportamento, um contínuo de programas e serviços (NJCLD, 2005). O objetivo principal do MRI é prevenir e solucionar as dificuldades académicas e de comportamento, proporcionando um ensino de sala de aula eficaz e intervenções de apoio de crescente intensidade e validadas pela investigação (FLETCHER; VAUGHN, 2009). Esse é um modelo que visa identificar e apoiar precocemente alunos que não progridem de forma adequada, ou seja, não respondem favorisco de apresentar dificuldades de aprendizagem específicas (FUCHS; FUCHS, 2007a).

No MRI destacam-se quatro componentes, nomeadamente: preTradução nossa, da expressão em inglês screening, school -wide screening ou universal screening. venção multinível; ${ }^{1}$ triagem universal; ${ }^{2}$ monitorização do progresso; ${ }^{3} \mathrm{e}$ análise de dados e tomada de decisão ${ }^{4}$ (Figura 1). 


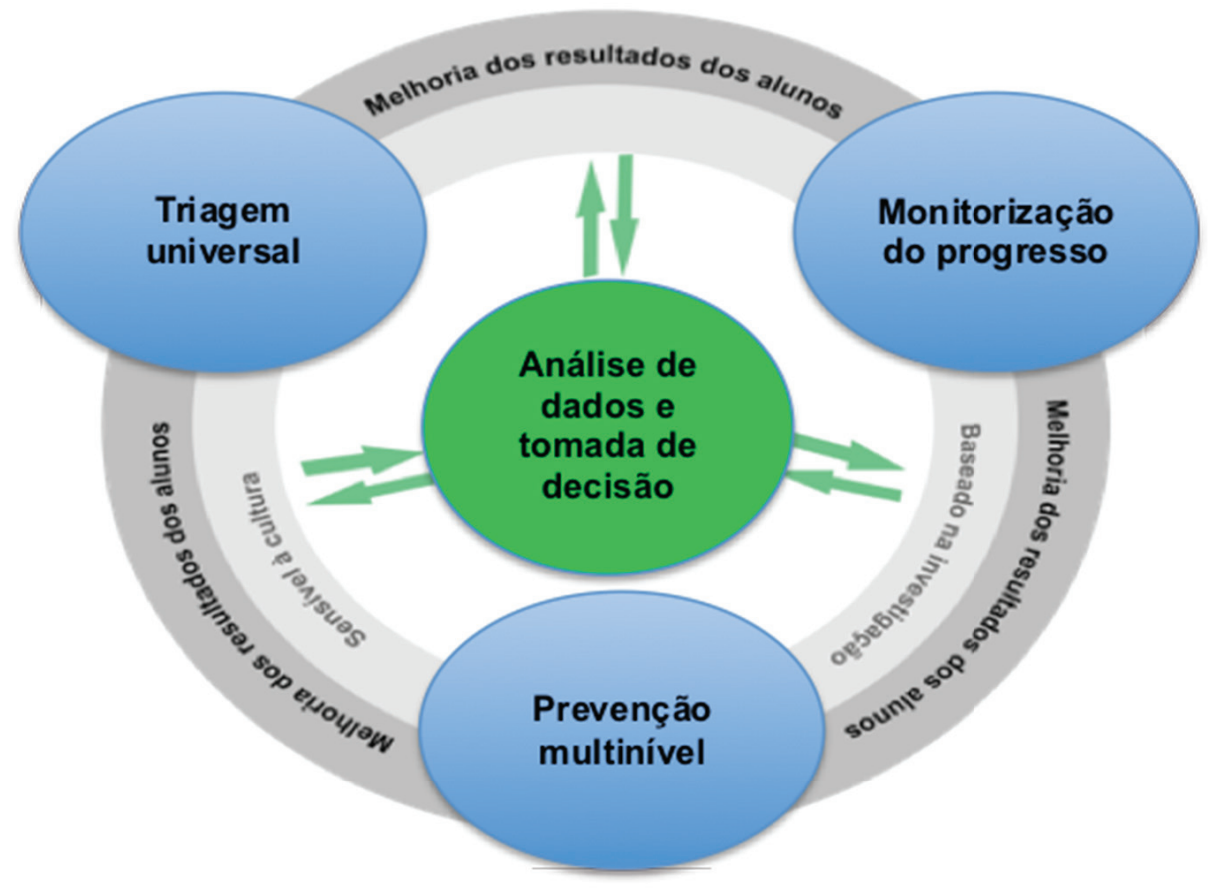

Fonte: Adaptada de National Center on Response to Intervention - NCRTI - (2012).

\section{PREVENÇÃO MULTINÍVEL}

O MRI é um sistema de prevenção, ensino e implementação de intervenções de apoio, desenvolvido em diferentes níveis (FLETCHER; VAUGHN, 2009; FUCHS; FUCHS, 2008; JOHNSON et al., 2006; NCRTI, 2012; NJCLD, 2005; VAUGHN; BOS, 2009) de intensidade ou prevenção (NATIONAL RESEARCH CENTER ON LEARNING DISABILITIES - NRCLD, 2007; NCRTI, 2012). Depois de identificados, os alunos têm a oportunidade de receber diferentes níveis de apoio/intervenção (geralmente três), sendo que apenas no caso de não responderem positivamente é que são encaminhados para os serviços de educação especial (TAYLOR; SMILEY; RICHARDS, 2009).

O nível 1 do modelo consiste na prevenção primária, o que significa proporcionar um ensino adequado de elevada qualidade, recorrendo ao uso de estratégias validadas pela investigação na classe regular, para todos os alunos, em cada ano de escolaridade, e pelo recurso a instrumentos de triagem com o objetivo de identificar alunos em risCO (BROWN-CHIDSEY; STEEGE, 2010; MARTINS; CORREIA; HALLAHAN, 2008-2009; NCRTI, 2012; NJCLD, 2005; NRCLD, 2007; TAYLOR, 2008; VAUGHN; BOS, 2009). Contudo, alguns alunos não têm sucesso mesmo recebendo um ensino adequado de alta qualidade, necessitando de uma intervenção adicional que vá ao encontro das suas características académicas e/ou de comportamento (VAUGHN; DENTON, 2008). Estes 
são alunos em risco (TAYLOR; SMILEY; RICHARDS, 2009), que transitam para o nível 2 de intervenção (VAUGHN; BOS, 2009).

No nível 2 desse modelo desenvolve-se prevenção secundária, ainda no contexto da classe regular. O professor de turma, ou um professor especializado, presta um apoio de moderada intensidade, individualmente ou em grupos pequenos, homogéneos, recorrendo a estratégias de intervenção baseadas na investigação (FUCHS; FUCHS, 2008; NCRTI, 2012; NJCLD, 2005; NRCLD, 2007; VAUGHN, 2003).

Já no nível 3 tem lugar uma intervenção individualizada (FUCHS; FUCHS, 2008; NCRTI, 2012; VAUGHN; BOS, 2009), intensa, sistemática (NJCLD, 2005) e especializada (NJCLD, 2005; NRCLD, 2007), realizada individualmente, em pares ou em grupos homogéneos (VAUGHN, 2003) de três alunos (VAUGHN; BOS, 2009). Esta intervenção é feita pelo professor do ensino regular (HAAGER; KLINGNER; VAUGHN, 2007; VAUGHN, 2003) ou por um professor especializado em intervir na área em que os alunos apresentam dificuldades (FLETCHER; VAUGHN, 2009; HAAGER; KLINGNER; VAUGHN, 2007; VAUGHN, 2003). Há, contudo, alunos no nível 3 que continuam a não responder à intervenção e, portanto, merecem um olhar ainda mais atento, no sentido de se identificarem, em concreto, suas dificuldades, que poderão traduzir-se na necessidade de apoio dos serviços de educação especial.

\section{TRIAGEM UNIVERSAL}

Uma outra componente do MRI é a triagem universal realizada no nível 1, que consiste na monitorização pontual de todos os alunos, no sentido de identificar ou sinalizar aqueles que estão em risco de apresentar resultados de aprendizagem baixos (FUCHS; FUCHS, 2008; NCRTI, 2012), de não adquirirem uma determinada capacidade importante e de, portanto, necessitarem de intervenção de nível 2 (JOHNSON et al., 2006). Tal processo tem como objetivo, também, providenciar uma intervenção o mais cedo possível, que promova resultados positivos e evite que o insucesso académico ou comportamental dos alunos se solidifique e os acompanhe em todo o seu percurso escolar e na vida adulta (FUCHS; FUCHS, 2008).

A triagem universal é feita em três momentos do ano letivo (início, meio e final) e incide sobre uma ou mais áreas, como a leitura, a escrita, a matemática, ou o comportamento (FUCHS; FUCHS, 2008; HUGHES; DEXTER, 2013; JOHNSON et al., 2006). Nesse processo são aplicadas, para todos os alunos, provas de realização breve, nomeadamente provas de monitorização com base no currículo - MBC - (NCRTI, 2012).

\section{MONITORIZAÇÃO DO PROGRESSO}

Transversal a cada um dos níveis do MRI destaca-se uma outra componente: a monitorização do progresso dos alunos (individualmente ou em grupo) com vista a avaliar o seu desempenho académico, no 
sentido de verificar de que forma eles se beneficiam do ensino na sala de aula, calcular a taxa de crescimento e de resposta à intervenção realizada e avaliar a eficácia dessa mesma intervenção, permitindo, consequentemente, perceber se há necessidade de efetuar ajustamentos à mesma (JOHNSON et al., 2006; NCRTI, 2012). A monitorização do progresso, no nível 1, funde-se com a triagem universal, enquanto nos níveis 2 e 3 objetiva perceber se a intervenção está a ter sucesso, ou seja, a ajudar os alunos a melhorar sua realização e a ter um crescimento adequado (JOHNSON et al., 2006).

A monitorização do progresso pode ser feita com recurso a provas de MBC (FUCHS; FUCHS, 2007b) que foram desenvolvidas na Universidade de Minnesota, por Deno e colaboradores, nos anos 1970 (SHINN; SHINN, 2002; STECKER; FUCHS; FUCHS, 2005; STECKER; LEMBKE; FOEGEN, 2008). Segundo Stecker, Sáenz e Lemons (2007), a MBC é um tipo de recolha de informação baseada no currículo nacional que, de acordo com Fuchs e Fuchs (2007b), de forma viável e tecnicamente forte, permite monitorizar o progresso dos alunos em diferentes áreas como leitura, expressão escrita, ortografia e matemática (BUSCH; LEMBKE, 2005; FUCHS; FUCHS, 2007b). A MBC é feita por meio de provas de realização breve e fáceis de administrar, cujas administração e cotação são realizadas de modo estandardizado (FUCHS; FUCHS, 2007b). Daqui resultam indicadores de proficiência global nas áreas académicas em análise (STECKER; LEMBKE; FOEGEN, 2008).

\section{ANÁLISE DE DADOS E TOMADA DE DECISÃO}

A análise de dados e a subsequente tomada de decisões que ocorrem nos três níveis da implementação do MRI são feitas com base na informação recolhida na triagem e na monitorização do progresso. São tomadas decisões acerca da necessidade de mudança de nível, de alteração das características da intervenção do nível e de encaminhamento para identificação de uma possível necessidade educativa especial (NCRTI, 2012).

A decisão referente à mudança de nível implica a definição de um critério que pode assumir diferentes especificidades. Na passagem do nível 1 para o nível 2, Deno et al. (2009) utilizam como critério o valor do percentil 20 dos resultados obtidos na triagem universal com a MBC, justificando que os $20 \%$ de alunos com resultados mais baixos, além de incluírem aqueles com maiores dificuldades, se traduzem num número de alunos (seis/sete por turma, aproximadamente) considerado razoável pelos professores para continuar a monitorizar individualmente.

Segundo Fuchs e Fuchs (2007a), a partir do nível 2 a mudança pode decorrer de quatro formas diferentes: estipulação de determinado valor de percentil (FUCHS; FUCHS, 2007a); consideração de um valor de referência para o final da intervenção ou do ano escolar (FUCHS; FUCHS, 
2007a); utilização da taxa de crescimento dos alunos durante a intervenção (FUCHS; FUCHS, 2007a); e emprego simultâneo do resultado final e do progresso dos alunos, identificando como não respondentes aqueles que, nesse contexto, estiverem pelo menos um desvio padrão abaixo dos restantes dos colegas (FUCHS et al., 2005).

Quando os alunos não respondem à intervenção no nível 3, a tomada de decisão prende-se com a identificação de dificuldades concretas. O facto de não responderem à intervenção no nível 3 não é ainda suficiente para se identificar, por exemplo, uma dificuldade de aprendizagem específica (NRCLD, 2007); implica, antes, uma avaliação compreensiva feita por uma equipa multidisciplinar (NRCLD, 2007), após autorização dos pais (NJCLD, 2005). Esta avaliação permitirá conhecer, compreender e identificar as dificuldades dos alunos e determinar a possível elegibilidade para os serviços de educação especial (BROWN-CHIDSEY; STEEGE, 2010; NRCLD, 2007).

Importa destacar que a transição entre níveis não é um processo unidirecional apenas no sentido ascendente, do nível 1 ao nível 3. Pelo contrário, gera-se no contexto do MRI toda uma dinâmica multinível, a partir da utilização trianual da MBC no nível 1, em que os alunos se movimentam entre os diferentes níveis em função dos seus problemas na aprendizagem (por exemplo na leitura) e da consecução dos objetivos considerados como aqueles que devem ser atingidos, tendo por base um critério previamente definido e que suporta o processo de tomada de decisão.

O MRI, cujas quatro componentes se descreveram, tem sido operacionalizado, implementado e pesquisado no sentido de se constituir como um modelo educativo que, por um lado, permite identificar e apoiar precocemente alunos que não progridem de forma adequada e que, assim, podem estar em risco académico e/ou comportamental e, por outro, torna-se uma forma de identificar alunos com dificuldades de aprendizagem específicas, ou seja, aqueles que não respondem a intervenção/ensino validados e estandardizados, necessitando, portanto, de uma intervenção individualizada dos serviços de educação especial (FUCHS; FUCHS, 2007a).

Nesse contexto, esboçou-se na presente investigação, para um agrupamento de escolas, uma nova forma de atuação em face dos problemas na leitura, tendo como pano de fundo a resposta dos alunos à intervenção e assentada em dois pilares essenciais: a triagem como base da prevenção, que se pretende multinível; e a monitorização do progresso recorrendo a provas de MBC. Assim, o presente estudo teve por finalidade analisar o uso de uma prova de MBC-Maze na triagem universal em alunos do $3^{\circ}$ ano de escolaridade do $1^{\circ}$ Ciclo do Ensino Básico - CEB - de um agrupamento de escolas no contexto do nível 1 de um MRI. Procurou-se conhecer: o nível de compreensão da leitura e a taxa 
de crescimento dos alunos; o impacto da variável risco no nível de compreensão da leitura e na taxa de crescimento dos alunos; e a fiabilidade dos resultados e validade concorrente da prova MBC-Maze.

\section{MÉTODO \\ POPULAÇÃO}

Nessa investigação participaram 82 alunos do $3^{\circ}$ ano do $1^{\circ}$ Ciclo do Ensino Básico de um agrupamento do norte de Portugal, sendo 47 $(57,3 \%)$ do género feminino e $35(42,7 \%)$ do género masculino. Esses alunos tinham oito anos de idade, não apresentavam necessidades educativas especiais - NEE - identificadas, nem usufruíam do apoio dos serviços de educação especial. Os participantes encontravam-se distribuídos por seis turmas, constituindo a população dos referidos ano e ciclo de ensino.

\section{INSTRUMENTOS DE RECOLHA DE DADOS}

Na presente investigação utilizaram-se dois instrumentos de recolha de dados que avaliam a compreensão da leitura: uma prova MBC-Maze (BROWN-CHIDSEY; DAVIS; MAYA, 2003; BUSCH; LEMBKE, 2005; DENO et al., 1989; SHINN; SHINN, 2002); e o teste de idade de leitura - TIL -, um teste estandardizado (SUCENA; CASTRO, 2010).

A adequação técnica da prova de MBC-Maze tem sido amplamente estudada, mostrando tratar-se de um instrumento válido e fiável para avaliar a leitura - ver, por exemplo, os estudos de Deno et al. (2009), Espin et al. (2010), Mercer et al. (2012), Pierce, McMaster e Deno (2010) e Tichá, Espin e Wayman (2009). De facto, recorre-se à MBC-Maze para monitorizar o nível de compreensão da leitura (BROWN-CHIDSEY; DAVIS; MAYA, 2003; BUSCH; LEMBKE, 2005; SHINN; SHINN, 2002), mas da sua administração e subsequente cotação resultam, também, indicadores gerais da realização dos alunos nesta área (BUSCH; LEMBKE, 2005). A MBC-Maze é, portanto, um indicador global do desempenho dos alunos na leitura e não algo que mede somente a compreensão, o que tem sido mostrado pelas suas elevadas correlações, quer com medidas de fluência, quer com outras medidas de compreensão da leitura (BUSCH; LEMBKE, 2005).

A prova de MBC-Maze foi previamente construída para ser utilizada nessa investigação, a partir de um texto de um manual escolar de língua portuguesa, do $3^{\circ}$ ano de escolaridade, que nunca tinha sido trabalhado pelos alunos. Uma vez escolhido o texto, procedeu-se à elaboração da prova, respeitando-se as regras enunciadas em Busch e Lembke (2005) e em Shinn e Shinn (2002). No texto, a primeira frase estava intacta e, a partir da segunda, cada sétima palavra foi substituída por três palavras apresentadas dentro de parênteses, sendo que uma delas - a palavra original - fazia sentido no texto e as outras duas não faziam 
sentido (distratores) (FUCHS; FUCHS, 2008; SHINN; SHINN, 2002). No total a prova construída apresentava 33 situações, sendo este o resultado máximo que cada aluno podia atingir. A prova foi realizada na sala de aula de forma coletiva (BUSCH; LEMBKE, 2005; PATRÃO, 2010; SHINN; SHINN, 2002) durante três minutos (BUSCH; LEMBKE, 2005; GRANEY et al., 2010; RICHARDSON; HAWKEN; KIRCHER, 2012; SHIN; DENO; ESPIN, 2000; SHINN; SHINN, 2002). A cotação da prova foi efetuada segundo o procedimento mais comum, que é o de terminar a cotação após o aparecimento de três erros consecutivos e contar o número de respostas corretas até o aparecimento desses três erros (WAYMAN et al., 2009).

Já o TIL avalia a leitura e pode ser usado para realização de um primeiro diagnóstico de dificuldades de leitura/dislexia. Durante a sua execução, o aluno lia silenciosamente frases isoladas, que não estavam completas, e escolhia uma das cinco palavras que se encontram dentro de parênteses para completar cada frase, sublinhando a palavra escolhida (SUCENA; CASTRO, 2010). Da prova fazem parte quatro frases para os alunos treinarem (presentes numa primeira página) e 36 frases experimentais (escritas na segunda página e distribuídas por duas colunas, de forma equitativa) (SUCENA; CASTRO, 2010). O tempo destinado à realização do TIL foi de cinco minutos e a cotação consistiu na soma das frases completadas corretamente, na multiplicação por 100 do número obtido e na divisão do produto resultante, por 36 (número total de frases) (SUCENA; CASTRO, 2010).

\section{PROCEDIMENTOS}

Depois de obtidas as devidas autorizações por parte da direção do agrupamento, dos professores e de todos os encarregados de educação, bem como a concordância dos alunos, agendaram-se as recolhas de dados durante o ano letivo. Assim, tiveram lugar, com a prova de MBC-Maze, uma primeira recolha no outono (no início de dezembro), uma segunda recolha já no inverno (a meio de março) e uma terceira recolha na primavera (final de maio). Com o TIL fez-se apenas uma recolha, que ocorreu na primavera (início de junho). A fiabilidade da administração das provas foi garantida por meio da verificação da manutenção, em todos os momentos de coleta de dados, dos procedimentos específicos relativos às instruções dadas aos alunos, às atividades de treino e ao limite de tempo. Assim, para assegurar que todos os procedimentos fossem igualmente repetidos, em todos os momentos foram preenchidas duas grelhas de validação para a prova MBC-Maze e para o TIL (VAZ, 2015). Ambos os documentos, preenchidos pelo professor da turma no decorrer de cada recolha de dados, tem o intuito de garantir a realização de todos os procedimentos necessários. Importa acrescentar que para a contagem do tempo de realização das provas foi utilizado sempre o mesmo cronómetro. 
Os procedimentos estatísticos utilizados foram de natureza descritiva: medidas de tendência central - média $(M)$, moda $(M o)$ e mediana (Me) -; valores mínimo e máximo; e uma medida de dispersão, o desvio padrão (DP), obtidos nas três monitorizações com a prova de MBC-Maze. Adicionalmente, usou-se estatística inferencial, nomeadamente o teste $t$ para amostras emparelhadas e independentes, o que permitiu testar a existência de diferenças estatisticamente significativas entre diferentes monitorizações (MAROCO, 2007; PESTANA; GAGEIRO, 2008). Finalmente, para o conhecimento da fiabilidade dos resultados e da validade concorrente da prova MBC-Maze, recorreu-se ao coeficiente de correlação Pearson ( $r$ de Pearson) calculado entre os resultados da primeira monitorização (outono) e os de um reteste realizado cerca de duas semanas após a mesma (ALMEIDA; FREIRE, 2000), e entre os resultados obtidos na prova MBC-Maze e aqueles encontrados no TIL, na primavera. Como se utilizaram grupos com mais de 30 participantes, o pressuposto da normalidade está implicitamente validado (MAROCO, 2007; PESTANA; GAGEIRO, 2008). Considerou-se um intervalo de confiança de 95\% para a diferença entre médias, sendo, portanto, de 5\% a probabilidade $(\mathrm{p} \leq .05)$ de obter um determinado resultado devido ao acaso.

\section{RESULTADOS}

Para responder aos objetivos do presente estudo, nessa secção apresentam-se os resultados da compreensão leitora e da taxa de crescimento semanal para os participantes, sublinha-se o impacto da variável risco e indicam-se a fiabilidade dos resultados e validade concorrente da prova MBC-Maze.

\section{NÍVEL DE COMPREENSÃO LEITORA E TAXA DE CRESCIMENTO SEMANAL PARA A POPULAÇÃO}

A Tabela 1 mostra o aumento da média de respostas corretas ao longo das três monitorizações. Assim, na possibilidade de um resultado máximo de 33 respostas corretas, a média mais baixa corresponde à monitorização do outono e a mais elevada refere-se àquela ocorrida na primavera, demonstrando que houve aprendizagem. Sublinhe-se que o desvio padrão aumenta entre as duas primeiras recolhas de dados, mas diminui na última, o que mostra que as diferenças entre os alunos se acentuam ao longo da primeira parte do ano letivo, mas diminuem no seu final. 
TABELA 1

RESULTADOS DESCRITIVOS DA POPULAÇÃO NA PROVA MBC-MAZE NAS TRÊS MONITORIZAÇÕES

\begin{tabular}{l|c|c|c|c|c|c|c}
\hline MONITORIZAÇÕES & N & M & DP & MO & ME & MÍNIMO & MÁXIMO \\
\hline Outono & 82 & 9.44 & 4.028 & $10^{*}$ & 9.00 & 1 & 22 \\
\hline Inverno & 82 & 14.65 & 6.251 & $8^{*}$ & 14.00 & 0 & 31 \\
\hline Primavera & 82 & 15.99 & 5.889 & $17^{*}$ & 16.00 & 1 & 32 \\
\hline
\end{tabular}

* Existem múltiplas modas. É apresentado o valor mais baixo.

Fonte: Elaboração dos autores.

O teste $t$ para amostras emparelhadas indica que existem diferenças estatisticamente significativas entre os resultados obtidos pela população nas monitorizações do outono e da primavera, com $t(81)=-15.8, p<0.001$. Isso significa que as diferenças entre essas monitorizações não se devem meramente ao acaso, remetendo para a existência de aprendizagem entre os períodos considerados, o que era de se esperar. Contudo, sublinha-se o facto de a média aumentar mais entre o outono e o inverno do que entre o inverno e a primavera.

Calculou-se a média das taxas de crescimento da população nos períodos entre: o outono e o inverno; o inverno e a primavera; e o outono e a primavera. Essas taxas foram obtidas por meio do cálculo do quociente resultante da diferença entre os resultados obtidos na segunda e na primeira monitorização e o número de semanas entre as duas monitorizações (BROWN; SKOW, 2009). Como se observa na Tabela 2, a taxa de crescimento semanal mais elevada ocorre entre a monitorização do outono e a do inverno.

TABELA 2

TAXAS MÉDIAS DE CRESCIMENTO ENTRE AS DIFERENTES MONITORIZAÇÕES

\begin{tabular}{l|c|c}
\hline MONITORIZAÇÕES & $\begin{array}{c}\text { TAXA MÉDIA DE } \\
\text { CRESCIMENTO }\end{array}$ & DP \\
\hline Outono-inverno & .40 & .33 \\
\hline Inverno-primavera & .13 & .37 \\
\hline Outono-primavera & .27 & .16 \\
\hline
\end{tabular}

Fonte: Elaboração dos autores.

Assim, considerando o ano letivo, é entre o outono e o inverno que os alunos que constituem a população mais crescem na aprendizagem dessa competência académica. Sublinhe-se que, no final do $3^{\circ}$ ano de escolaridade, a média de resultados das raparigas é superior à dos rapazes ( $M=16.23$ e $M=15.66$, respetivamente), bem como a taxa de crescimento semanal durante o ano letivo (.28 e .26, respetivamente), embora as diferenças não sejam estatisticamente significativas. Isso quer dizer, então, que as diferenças encontradas não se traduzem em mais aprendizagem de um ou de outro género, sendo provavelmente diferenças ocasionais. 


\section{IMPACTO DA VARIÁVEL RISCO NO NÍVEL DE COMPREENSÃO LEITORA E NA TAXA DE CRESCIMENTO SEMANAL}

A identificação dos alunos em risco na população, em cada umas das três monitorizações, teve por base o percentil 20 da mesma, tal como preconizado por Busch e Lembke (2005). Assim, verificou-se que há dez alunos que se mantêm em risco nas três monitorizações, ou seja, durante todo o ano letivo. Esses dez alunos correspondem a 12,2\% da população e a 58,8\% dos 17 alunos identificados em risco no outono quando se leva em conta o percentil 20 da população. Considerando-se os dez alunos em risco ao longo de todo o ano letivo, verifica-se ainda que dois deles estão sempre no percentil 15 e quatro estão sempre no percentil 10 .

Os resultados apresentados na Tabela 3 mostram a realização e a evolução na leitura dos alunos que estão em risco nas sucessivas monitorizações do ano letivo e daqueles que nunca foram identificados em risco em nenhuma das monitorizações.

\section{TABELA 3}

DESCRIÇÃO ESTATÍSTICA DOS RESULTADOS NA PROVA MBC-MAZE DOS ALUNOS EM RISCO NO ANO LETIVO E DOS ALUNOS QUE NUNCA ESTIVERAM EM RISCO

\begin{tabular}{l|c|c|c|c|c|c|c}
\hline MONITORIZAÇÕES & N & M & DP & MO & ME & MÍNIMO & MÁXIMO \\
\hline Outono & & & & & & & \\
\hline Em risco & 10 & 4.10 & 2.025 & $5^{*}$ & 5.00 & 1 & 6 \\
\hline Sem risco & 53 & 11.25 & 3.322 & 11 & 11.00 & 7 & 22 \\
\hline Inverno & & & & & & & \\
\hline Em risco & 10 & 5.70 & 3.057 & 8 & 6.50 & 0 & 9 \\
\hline Sem risco & 53 & 17.68 & 5,121 & 16 & 16.00 & 11 & 31 \\
\hline Primavera & & & & & & & \\
\hline Em risco & 10 & 8.30 & 1.703 & 7 & 8.00 & 6 & 11 \\
\hline Sem risco & 53 & 18.91 & 4.732 & 18 & 18.00 & 12 & 32 \\
\hline
\end{tabular}

Fonte: Elaboração dos autores.

Nas monitorizações do outono e da primavera, os alunos que nunca estiveram em risco apresentam médias acima do dobro daquelas observadas para os alunos que estiveram sempre em risco. Na monitorização do inverno os alunos que nunca estiveram em risco têm uma média superior ao triplo daquela obtida pelos alunos que estiveram sempre em risco. Comparando, em cada monitorização, a média dos alunos que estão em risco nas três monitorizações do ano letivo com a média dos alunos que nunca estiveram em risco, verifica-se que a diferença aumenta entre a monitorização do outono e a do inverno (7.15 e 11.98, respetivamente). Essa diferença é um pouco inferior na monitorização da primavera, relativamente à segunda monitorização (10.61), embora seja ainda superior à diferença encontrada na monitorização do outono. Isso significa, então, que os alunos sem risco e aqueles que se encontram em risco durante todo o ano letivo se distanciam ao longo desse período, sendo no inverno e na primavera que se encontram mais 
distantes, embora na primavera um pouco menos do que no inverno. Tais resultados remetem, assim, para o facto de estes alunos, não sendo detetados logo no início do ano, verem acentuar-se suas dificuldades e a distância que os separa dos alunos que nunca apresentam risco, ou seja, dos bons leitores, o que evidencia uma necessidade de atuação por parte do sistema de ensino para contrariar essa situação.

Para os mesmos alunos calcularam-se as taxas de crescimento nos diferentes períodos: outono-inverno; inverno-primavera; e outono-primavera (Tabela 4).

TABELA 4

TAXAS MÉDIAS DE CRESCIMENTO DOS ALUNOS EM RISCO E DAQUELES QUE NUNCA ESTIVERAM EM RISCO NAS TRÊS MONITORIZAÇÕES DO ANO LETIVO

\begin{tabular}{l|c}
\hline MONITORIZAÇÕES & $\begin{array}{c}\text { TAXAS MÉDIAS DE } \\
\text { CRESCIMENTO }\end{array}$ \\
\hline Outono-inverno & .12 \\
\hline Em risco & .49 \\
\hline Sem risco & \\
\hline Inverno-primavera & .26 \\
\hline Em risco & .13 \\
\hline Sem risco & .18 \\
\hline Outono-primavera & .32 \\
\hline Em risco & \\
\hline Sem risco & \\
\hline
\end{tabular}

Fonte: Elaboração dos autores.

Os resultados mostram que, no período outono-inverno, os alunos sem risco apresentam maior taxa de crescimento, situação que se inverte no período inverno-primavera, em que a taxa de crescimento é maior para os alunos em risco. Considerando-se as diferentes monitorizações do ano letivo, os alunos em risco alcançaram maior taxa entre as monitorizações do inverno e da primavera do que entre aquelas realizadas no outono e no inverno, acontecendo o contrário com os alunos que nunca estiveram em risco. Tendo por base o ano letivo na sua globalidade, ou seja, o crescimento entre o outono e a primavera, são os alunos que nunca estiveram em risco que mais evoluem.

Interessa ainda sublinhar que, considerando o período outono-inverno, os alunos sem risco apresentam taxa de crescimento quatro vezes superior à dos alunos em risco. Essa situação altera-se no período inverno-primavera, em que a taxa dos alunos em risco nas três monitorizações é o dobro daquela dos alunos sem risco. Assim, talvez tenha existido por parte do professor uma maior atenção entre o inverno e a primavera aos alunos que entre o outono e o inverno evidenciaram problemas na leitura. Os alunos que nunca estiveram em risco, sendo bons leitores e estando no $3^{\circ}$ ano de escolaridade, evidenciam cada vez menos crescimento porque efetivamente eles já sabem ler, apenas aperfeiçoam a partir daí a sua leitura. 


\section{FIABILIDADE DOS RESULTADOS E VALIDADE CONCORRENTE DA PROVA MBC-MAZE}

O $r$ de Pearson calculado com os resultados da primeira monitorização (outono) e de um reteste realizado cerca de duas semanas após a mesma apresenta o valor de .780, significativo a $1 \%$ e indicativo de uma associação linear alta (WAYMAN et al., 2007). Estudou-se ainda a validade concorrente dos resultados por meio do cálculo do $r$ de Pearson entre os resultados obtidos na prova Maze na monitorização da primavera e aqueles do teste de idade de leitura (SUCENA; CASTRO, 2010), obtendo-se o valor de .780 também significativo a $1 \%$.

\section{CONCLUSÕES}

O estudo que aqui se apresenta foi dedicado à investigação sobre o risco na leitura, durante o qual muitas dúvidas surgiram, outras tantas foram sendo esclarecidas e inúmeras ficaram sem resposta. No contexto da investigação lançou-se um novo olhar sobre esses alunos que, nos primeiros anos da sua escolaridade, se confrontam com problemas na leitura e veem para sempre fechadas as portas de um mundo novo, o mundo do conhecimento que é permitido pelo prazer de ler um livro. E a partir desse novo olhar, esboça-se uma nova forma de atuação assente na prevenção e na deteção precoce dos seus problemas na leitura. Assim, o estudo quantitativo que tem sido apresentado nesse artigo conduziu a algumas conclusões esboçadas a seguir.

Os resultados mostram que a aprendizagem da compreensão leitora ao longo do ano letivo é feita com uma taxa de crescimento semanal de .27. No final do $3^{\circ}$ ano de escolaridade, a média de resultados da população é de 15.99 ( $D P=5.889)$ e a taxa de crescimento semanal durante o ano letivo é de .27 ( $D P=.16)$. Ao longo das diferentes monitorizações, os alunos aumentam o valor de média dos seus resultados, situação também encontrada por Deno et al. (2009) e por Pierce, McMaster e Deno (2010), o que, além de desejável, é de se esperar uma vez que mostra a existência de progressão dos alunos ao longo do ano letivo. As diferenças encontradas entre as médias obtidas na monitorização do outono e na da primavera são estatisticamente significativas tal como se verifica em Pierce, McMaster e Deno (2010), significando que a progressão dos alunos traduz efetivamente aprendizagem entre o início e o final do ano letivo.

Os alunos que constituem a população apresentam maior progresso entre o outono e o inverno do que entre o inverno e a primavera. Tal observação vai ao encontro do que constataram Ardoin e Christ (2008), Christ et al. (2010) e também Nese et al. (2012), que, aliás, verificaram esta situação de forma mais evidente em alunos do $3^{\circ}$ ano de escolaridade. 
De acordo com Christ et al. (2010), esse efeito sazonal pode ficar a dever-se a alterações nas condições de ensino e na motivação dos alunos à medida que se caminha para o final do ano letivo. Também nesse sentido é apontado por Good, Cooper e Blakey (1980) o facto de o professor influenciar mais os alunos no início do ano (outono) do que no inverno e na primavera, por exemplo, por meio do elogio ao seu comportamento. Assim, parece legítimo pensar que essa situação pode conduzir a níveis de motivação inferiores na primavera e, consequentemente, a um menor avanço dos alunos. O efeito sazonal pode ainda estar relacionado com o facto de os professores investirem mais na organização e gestão da aula no início do ano letivo (CAMERON; CONNOR; MORRISON, 2005). Assim, será importante que o professor mantenha ao longo de todo o ano letivo a postura que o carateriza no início, continuando a praticar o elogio e a investir na organização e gestão da sala de aula. Pode acontecer também que os alunos no início do $3^{\circ}$ ano estejam ainda a ganhar e a solidificar competências de leitura e que, nesse contexto, o seu progresso seja mais evidente. À medida que tais competências vão sendo adquiridas, o aluno passa a ler e sua evolução torna-se menos visível.

Nessa investigação, observou-se que dez alunos estavam em risco na leitura durante todo o ano letivo. Na primeira monitorização, feita no outono, 17 alunos foram considerados em risco, dos quais dez $(58,8 \%)$ mantiveram-se em risco durante todo o ano letivo. Significa então que, para mais de metade dos alunos que foram identificados em risco no outono, o sistema de ensino não atuou no sentido de prevenir a manutenção ou o agravamento das dificuldades que estes alunos apresentam.

No final do $3^{\circ}$ ano de escolaridade, a média de resultados dos alunos que nunca estiveram em risco é o dobro daquela referente aos alunos que estiveram em risco todo o ano letivo ( $M=18.91$ e $M=8.30$, respetivamente). Tal diferença regista-se também na monitorização do outono, sendo ainda maior naquela realizada no inverno, com os alunos que nunca estiveram em risco a apresentarem uma média que é mais do que o triplo da média dos alunos em risco no ano letivo.

Situação idêntica encontraram Deno et al. (2009), ao observarem no outono um resultado mais do que três vezes superior para os alunos sem risco em relação àqueles em risco e, no inverno e na primavera, resultados para os alunos sem risco superiores ao dobro dos resultados dos alunos em risco.

Importa ainda destacar que os alunos que se mantiveram em risco durante todo o ano letivo ficaram menos distantes dos colegas que nunca estiveram em risco na monitorização do outono e mais distantes nas monitorizações do inverno e da primavera. Aliás, esses alunos distanciaram-se sobretudo entre o outono e o inverno, período em que os alunos que nunca estiveram em risco obtiveram taxa de crescimento aproximadamente quatro vezes maior do que os alunos em risco nas 
três monitorizações do ano letivo, o que significa que, se o sistema não atuar precocemente num momento inicial do ano letivo, os alunos em risco ficam cada vez mais distantes dos colegas sem risco, com as respetivas repercussões que esta situação tem ao nível do seu sucesso escolar. Acentua-se assim a necessidade de uma atuação precoce e preventiva com vista a evitar ao máximo o agravamento dos problemas na leitura dos alunos que os apresentam.

A taxa de crescimento semanal dos alunos que nunca estiveram em risco é aproximadamente o dobro daquela obtida pelos alunos que estiveram em risco durante todo o ano letivo (.32 e .18, respetivamente). Essa diferença corrobora os resultados de Deno et al. (2009), que verificaram que os alunos em risco apresentaram menores taxas de crescimento durante o ano letivo.

Os alunos em risco durante o ano letivo alcançaram mais progresso entre o inverno e a primavera, com taxa de crescimento maior do que a dos alunos que nunca estiveram em risco. Já estes últimos tiveram mais progresso entre o outono e o inverno. Tal situação pode ficar a dever-se ao facto de as dificuldades dos alunos que estão em risco em todas as monitorizações do ano letivo se tornarem evidentes entre o outono e o inverno, podendo haver em seguida um olhar mais atento e um maior investimento no dia-a-dia por parte do professor. Este, ao aperceber-se dos problemas dos alunos na leitura, na parte inicial do ano letivo, talvez esteja a apoiar/investir um pouco mais nesses alunos entre o inverno e o final do ano letivo, ainda que não se trate de um apoio organizado sob a forma de intervenção formal. E os próprios alunos estarão possivelmente a ter consciência dos seus problemas, o que os leva provavelmente a esforçarem-se para os ultrapassar. Já os alunos que nunca se encontram em risco evoluem na leitura sobretudo até o inverno, passando essa evolução a ser menor a partir desse período porque, efetivamente, sendo alunos que nunca estiveram em risco, leem bem e, a partir desse momento, seu progresso é menor, o que vai ao encontro do referido por Christ et al. (2010), Deno et al. (2001) e Nese et al. (2012).

Tais resultados apontam para uma necessidade urgente de atuação por parte do professor e do atual sistema de ensino perante os problemas na leitura dos alunos em risco, no sentido de inverter um percurso indubitável em direção ao insucesso. Adicionalmente, assumem grande importância para o agrupamento em questão, que poderá dispor dos resultados obtidos para servirem de referência nas tomadas de decisão dos professores do $3^{\circ}$ ano de escolaridade. Assim, tendo por base as conclusões desse estudo, bem como o modelo teórico que o fundamenta, propõe-se para este agrupamento o uso dos valores do percentil 20 e do nível e da taxa de compreensão leitora dos participantes (que constituíam a população) como referência para a realização de intervenções de prevenção primária e secundária para os alunos identificados 
como estando em risco e a sua monitorização semanal. Um cuidado especial deve ser tido para os alunos no ou abaixo do percentil 10.

A presente investigação pretendeu responder a uma lacuna que existe no sistema educativo português no campo dos problemas na leitura e mais especificamente das dificuldades de aprendizagem específicas na leitura, ao apresentar uma nova forma de atuação que se constitui como um modelo preventivo, em que a MBC, as provas Maze e o estudo do risco assumem um lugar de destaque por permitirem identificar precocemente alunos em risco e assim desencadear um processo interventivo logo que os seus problemas na leitura começam a manifestar-se.

\section{REFERÊNCIAS}

ALMEIDA, L. S.; FREIRE, T. Metodologia da investigação em psicologia e educação. Braga: Psiquilíbrios, 2000 .

ARDOIN, S. P.; CHRIST, T. J. Evaluating curriculum-based measurement slope estimates using data from triannual universal screenings. School Psychology Review, v. 37, n. 1, p. 109-125, 2008.

BROWN-CHIDSEY, R.; DAVIS, L.; MAYA, C. Sources of variance in curriculum-based measures of silent reading. Psychology in the Schools, v. 40, n. 4, p. 363-377, 2003.

BROWN-CHIDSEY, R.; STEEGE, M. W. Response to intervention: principles and strategies for effective practice. New York: Guilford Press, 2010.

BROWN, J.; SKOW, K. RTI: data-based decision making. The Iris Center, 2009.

BUSCH, T. W.; LEMBKE, E. S. Teaching tutorial 5: progress monitoring in reading using the CBM maze procedure. Charlottesville, VA: Division for Learning Disabilities of the Council for Exceptional Children, 2005.

CAMERON, C. E.; CONNOR, C. M.; MORRISON, F. J. Effects of variation in teacher organization on classroom functioning. Journal of School Psychology, v. 43, n. 1, p. 61-85, 2005.

CHRIST, T. J. et al. Curriculum-based measurement of oral reading: an evaluation of growth rates and seasonal effects among students served in general and special education. School Psychology Review, v. 39, n. 3, p. 447-462, 2010.

DENO, S. L. et al. Basic academic skill samples: instructions for administration and skill samples. Minnesota: U.S. Department of Education Grant, 1989.

DENO, S. L. et al. Using curriculum-based measurement to establish growth standards for students with learning disabilities. School Psychology Review, v. 30, n. 4, p. 507-524, 2001.

DENO, S. L. et al. Developing a school-wide progress-monitoring system. Psychology in the Schools, v. 46 , n. 1, p. 44-55, 2009.

ESPIN, C.; WALLACE, T.; LEMBKE, E.; CAMPBELL, H.; LONG, J. D. Creating a progress-monitoring system in reading for middle-school students: Tracking progress toward meeting high-stakes standards. Learning Disabilities Research \& Practice, v. 25, n. 2, p. 60-75, 2010.

FLETCHER, J. M.; VAUGHN, S. Response to intervention: preventing and remediating academic difficulties. Child Development Perspectives, v. 3, n. 1, p. 30-37, Apr. 2009.

FUCHS, L. S. et al. The prevention, identification, and cognitive determinants of math difficulty. Journal of Educational Psychology, v. 97, n. 3, p. 493-513, 2005.

FUCHS, L. S.; FUCHS, D. A model for implementing responsiveness to intervention. Teaching Exceptional Children, v. 39, n. 5, p. 14-20, 2007a.

FUCHS, L. S.; FUCHS, D. Using CBM for progress monitoring in reading. Washington, DC: United States Office of Special Education Program/Student Progress Monitoring, 2007b. 
FUCHS, L. S.; FUCHS, D. The role of assessment within the RTI framework. In: FUCHS, D. et al. (Ed.). Response to intervention: a framework for reading educators. Newark: International Reading Association, 2008. p. 27-49.

GOOD, T. L.; COOPER, H. M.; BLAKEY, S. L. Classroom interaction as a function of teacher expectations, student sex, and time of year. Journal of Educational Psychology, v. 72, n. 3, p. 378-385, 1980.

GRANEY, S. B. et al. Universal screening of reading in late elementary school: R-CBM versus CBM maze. Remedial and Special Education, v. 31, n. 5, p. 368-377, 2010.

HAAGER, D.; KLINGNER, J.; VAUGHN, S. Evidence-based reading practices for response to intervention. Baltimore: Paul H. Brookes, 2007.

HUGHES, C.; DEXTER, D. D. Universal screening within a response-to-intervention model. New York: RTI Action Network, 2013. Disponível em: <http://www.rtinetwork.org/learn/research/ universal-screening-within-a-rti-model>. Acesso em: 18 out. 2013.

JOHNSON, E. et al. Responsiveness to intervention (RTI): how to do it. Lawrence: National Research Center on Learning Disabilities, 2006.

MAROCO, J. Análise estatística com utilização do SPSS 3. Lisboa: Sílabo, 2007.

MARTINS, A. P. L.; CORREIA, L. M.; HALLAHAN, D. Compreender o fenômeno das dificuldades de aprendizagem a partir de sete estudos de caso: conclusões de um estudo naturalista. Inclusão, n. 8, p. 19-48, 2008-2009.

MERCER, S. H. et al. Generalizability theory analysis of CBM maze reliability in third-through fifth-grade students. Assessment for Effective Intervention, v. 37, n. 3, p. 183-190, 2012.

NATIONAL CENTER ON RESPONSE TO INTERVENTION. Multi-level Prevention System. New York: NCRI, 2012. Disponível em: <http://www.rti4success.org/categorycontents/multi-level_ prevention_system>. Acesso em: 1 jan. 2012.

NATIONAL JOINT COMMITTEE ON LEARNING DISABILITIES. Responsiveness to intervention and learning disabilities. Arlington, VA: NJCLD, 2005.

NATIONAL RESEARCH CENTER ON LEARNING DISABILITIES. What is responsiveness to intervention? Lawrence: NRCLD, 2007.

NESE, J. F. T. et al. Within-year oral reading fluency with CBM: a comparison of models. Reading and Writing, v. 25, n. 4, p. 887-915, 2012.

PATRÃO, M. S. G. Monitorização com base no currciculo: um estudo quantitativo sobre a utilização de provas maze no contexto do nível I do modelo de atendimento à diversidade. Dissertação (Mestrado) - Universidade do Minho, Braga, 2010.

PESTANA, M. H.; GAGEIRO, J. N. Análise de dados para ciências sociais: a complementaridade do SPSS. Lisboa: Sílabo, 2008.

PIERCE, R.; McMASTER, K.; DENO, S. L. The effects of using different procedures to score maze measures. Learning Disabilities Research \& Practice, v. 25, n. 3, p. 151-160, 2010.

RICHARDSON, R. D.; HAWKEN, L. S.; KIRCHER, J. Bias using maze to predict high-stakes test performance among hispanic and spanish-speaking students. Assessment for Effective Intervention, v. 37, n. 3, p. $159-170,2012$.

SHIN, J.; DENO, S. L.; ESPIN, C. Technical adequacy of the maze task for curriculum-based measurement of reading growth. Journal of Special Education, v. 34, n. 3, p. 164-172, Fall 2000.

SHINN, M. R.; SHINN, M. M. AIMSweb training workbook: administration and scoring of reading maze for use in general outcome measurement. Eden Prairie, MN: Edformation, 2002.

STECKER, P. M.; FUCHS, L. S.; FUCHS, D. Using curriculum-based measurement to improve student achievement: review of research. Psychology in the Schools, v. 42, n. 8, p. 795-819, 2005.

STECKER, P. M.; LEMBKE, E. S.; FOEGEN, A. Using progress-monitoring data to improve instructional decision making. Preventing School Failure, v. 52, n. 2, p. 45-58, Winter 2008. 
STECKER, P. M.; SÁENZ, L.; LEMONS, C. Introduction to using CBM for progress monitoring in reading. Summer Institute on Student Progress Monitoring, 2007.

SUCENA, A.; CASTRO, S. L. Aprender a ler e avaliar a leitura. O TIL: teste de idade de leitura. Coimbra: Almedina, 2010.

TAYLOR, B. M. Tier 1: Effective classroom reading instruction in the elementary grades. In: FUCHS, D. et al. (Ed.). Response to intervention: a framework for reading educators. Newark: International Reading Association, 2008. p. 5-25.

TAYLOR, R. L.; SMILEY, L.; RICHARDS, S. B. Estudiantes excepcionales: formación de maestros para el siglo XXI. New York: McGraw-Hill, 2009.

TICHÁ, R.; ESPIN, C.; WAYMAN, M. M. Reading progress monitoring for secondary-school students: reliability, validity, and sensitivity to growth of reading-aloud and maze-selection measures. Learning Disabilities Research \& Practice, v. 24, n. 3, p. 132-142, 2009.

VAUGHN, S. How many tiers are needed for response to intervention to achieve acceptable prevention outcomes? In: NATIONAL RESEARCH CENTER ON LEARNING DISABILITIES RESPONSIVENESS-TO-INTERVENTION SYMPOSIUM, 2003. Kansas City: NRC, 2003.

VAUGHN, S.; BOS, C. Strategies for teaching students with learning and behavior problems. 7. ed. New Jersey: Pearson, 2009.

VAUGHN, S.; DENTON, C. A. Tier 2: the role of intervention. In: FUCHS, D. et al. (Ed.). Response to intervention: a framework for reading educators. Newark: International Reading Association, 2008. p. $51-70$

VAZ, P. M. F. Triagem universal de alunos em risco de apresentarem dificuldades de aprendizagem específicas na leitura: um estudo quantitativo no $3^{\circ}$ ano do $1^{\circ}$ ciclo do ensino básico. Tese (Doutorado em Educação) - Universidade do Minho, Braga, 2015.

WAYMAN, M. M. et al. Literature synthesis on curriculum-based measurement in reading. Journal of Special Education, v. 41, n. 2, p. 85-120, Summer 2007.

WAYMAN, M. M. et al. Comparison of different scoring procedures for the CBM maze selection measure. Minnesota: University of Minnesota, Reasearch Institute on Progress Monitoring, 2009.

\section{PAULA MARISA FORTUNATO VAZ}

Escola Superior de Educação do Instituto Politécnico de Bragança, Bragança, Portugal

paulavaz@ipb.pt

\section{ANA PAULA LOUÇÃO MARTINS}

Centro de Investigação em Educação, Instituto de Educação da Universidade do Minho, Minho, Portugal

apmartins@ie.uminho.pt

\section{LUÍS DE MIRANDA CORREIA}

Professor Catedrático Aposentado do Instituto de Educação da Universidade do Minho, Minho, Portugal

lmiranda@ie.uminho.pt 